
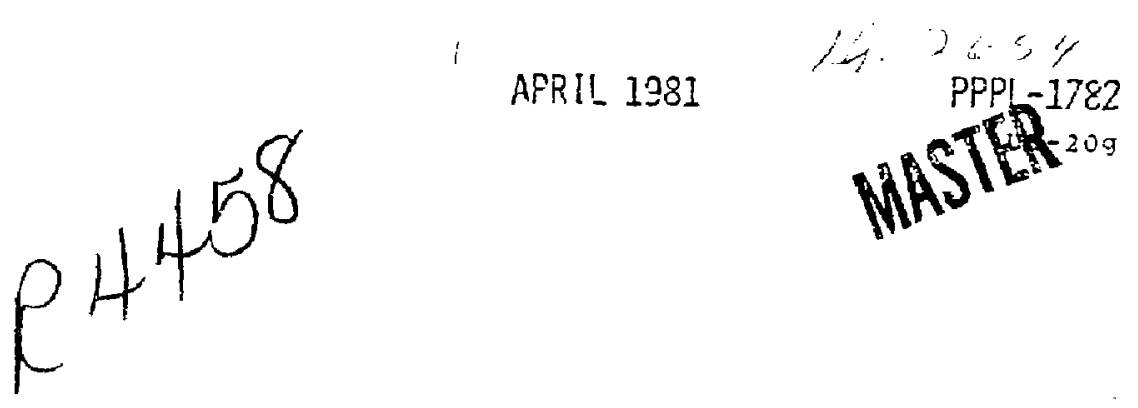

\title{
KINETIC THEORY OF COLLISIONLESS BALLOONING MODES
}

BY

C. Z, EUENG

\section{PLASMA FHYSICS ZABORATORY}

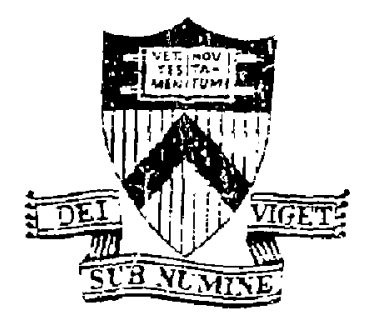

\section{PRINCETON UNIVERSITY PRINCETON, NEW JERSEY}

This work suboorted by the U.S. Nepartmenc of snergy Concract its. OE-AC52-73-CHO-3073. Reproduction, translation, ouglicetion, use and disposal, in whole or in part, ov or for the Jrited 5 tates acvernmert is farnicted.' 


\section{Kinetic Theury of Collisionless Ballooning Modes}

\section{by}

\section{C. $z$. Cheng}

Piasma Physics Laboratory, Prfnceton University

Princeton, New Jersey 08544

ABSTRACT

A kinetic ballooning mode equation retaining full finlte ion Larmor radius and ion magnetic drift resonance effects is derived by employing the high $n$ ballooing mode formalism. We find that the critical $B$ is smaller than the ideal MHD critical $B$, except when $\eta_{i}=0\left(\eta_{i} \equiv d e n T_{i} / d R n N\right)$ they are identical. The finite Latmor radius effects reduce the growth rate but io noc stabilize the mode. The ion magnetle drift resonance effects are destabilizing. 


\section{INTRODUCTION}

It is believed that the plasma confinement in hfgh temperature axisymmetric tokamaks may be 1 imited by the instability of magnetohydrodynami? (MHD) ballooning modes. According to ldeal MHD theory the ballooning. modes can be driven unstable ty the combined effects of the magnetic curvature and the pressure gradient. When $B$ (the ratio of the kinetic pressure to the magnetic pressure) increases to a cricical value, $B_{c}$, an unstable mode can deveiof and balloons in the bad curvature region. Many calculations l-5 have been done to determine $B_{c}$ from the ideal MHD equations. One of the basir assumptions of ideal MHD theary is that the paratlel electric field perturbation $E_{\|}$vanishes. However, $E_{i \mid}$ may become finite when kinetic effects such as finite Larmor radius, magnetic :rift and Landau resonances, trapped particles and collisional effects are included. Especially, in the high $n$ limit these kinetic effects can be signi:-1cant. It is, therefore, of interest to examine whether these kinetic effects could modify the stability of the balloontne modes and hence $B_{C}$.

Previous investigations ${ }^{6,7}$ of the kinetic effects on the Mid ballooning modes have made use of the assumption that the ion magnetir. irift trequency, ${ }^{\omega}$ Di, is small compezed with the mode frequency, $\omega$, and is treated perturbatively without Including resonances. However, this assumption is not always valid, especially when the modes are near marginal stability. In this paper we will remove this limitation and Investigate the stability of the ballooning modes by retaining full finite ton larmor radius and ion magnetic drift resonance effects. Effects due to trapped particles, Lanjau resonances, and col11sons will be ignored. 
Employing the high $\mathrm{n}$ ballooning mode and WKB formalism, ${ }^{-5}$ we have derived, in Section II, a set of elgenmode equations governing both drift and shear Alfuén waves. If terms of order $\left(\omega_{t i} / \omega\right)^{2}$ are neglected $\left(\omega_{t i}\right.$ is the ion transit frequency), we can obtain a single second order differential equation describing the kinetic ballooning modes. Then, we establish its relationship to the ideal MHD baliooning mode equation. In Section III, we first observe that for $\eta_{i}=0\left(\eta_{1} \equiv \mathrm{d} \ell \mathrm{nT}{ }_{1} / \mathrm{d} \ell \mathrm{nN}\right)$ the kinetfe ballooning mode equation reduces to the ideal MHD ballooning mode equation at marginal stabflity with $\omega=\omega_{*_{i}}$ $\left(\omega_{*_{i}}\right.$ is the ion diamagnetic drift frequency). This means that $\beta_{c}$ is identical to the ideal $M H D B_{C}$ and is fndependent of the lon Larmor radius and magnetic drift resonance effects. For $n_{i} \neq 0, B_{c}$ is found to be smaller than the ideaI MHD $3_{c}$, and numerical solutions with parametric variations of $B$, etc. are presented in detail. rinally, a summary is given in section IV.

\section{FORMULATION}

Let us consider low B plasma in an axisymmetrie, large aspect ratio corus with concentric, circular magnetic surfaces. We use a $(r, \partial, \zeta)$ coordinate system, where $r$ is the minor radius, $\theta$ is the poloidal angle, and $b$ is the tornidal angle. The equilibrium magnetic field is given by $\vec{B}=B_{0}$ $(1-r \cos \theta / R)(\hat{\zeta}+(r / q R) \hat{\theta})$. The perturbed quantitles can be expressed in the form

$$
\Phi=\phi(r, \theta) \exp [1(m \theta-n \zeta-\omega t)]
$$

The I Inearized lon gyrokinetce equation is given by 


$$
\begin{aligned}
& {\left[\omega-\omega_{t i} \hat{v}_{\|}\left(s-1 \frac{\partial}{\partial \theta}\right)+\hat{\omega}_{d i}\right\} H_{1}} \\
& \quad=\left[\frac{e F_{M}}{T}\right)_{1}\left\{\omega-\omega_{\star_{i}}\left[1+\eta_{1}\left(\hat{v}^{2}-\frac{3}{2}\right)\right] J_{0}\left(\frac{k_{1} v_{1}}{\Omega_{1}}\right)\left(\Phi-v_{n} A_{n} / e\right)\right.
\end{aligned}
$$

where $w_{t i}=v_{1} / q R$ is the ion transit frequency, $s=\left(r-r_{0}\right) / \Delta r_{s}, r_{0}$ is the minor radius of the reference mode rational surface with $m=n q\left(r_{0}\right)$, $\Delta r_{s}=1 / k_{\theta} \hat{s}, k_{\theta}=m / r_{0}, \hat{s}=\left(r q^{\prime} / q\right)$ at $r=r_{0}, \vec{k}_{1}=-1[(\hat{\theta} / r) \partial / \partial \theta+\hat{r} \partial / \partial r], \Omega_{i}$ $=e B / m_{i} c, \eta_{i}=\left(d \ell n T_{i} / d \ell n N\right)$, the magnetic drift frequency is $\omega_{d i} \cong\left(2 \varepsilon_{n} / T\right)$ $\omega_{*_{e}}\left(\hat{v}_{\|}^{2}+\hat{v}_{1}^{2} / 2\right)(\cos \theta-i \hat{s} \sin \theta \partial / \partial s) \tau=T_{e} / T_{1}, E_{n}=r_{n} / R, r_{n}$ ls the density scale length, $R$ is the major radius of the torus, $q$ is the safety factar, $\omega_{\star_{e}}=\left(c T_{e} / e B\right)\left(k_{g} / r_{n}\right), \omega_{\star_{i}}=-\omega_{\star_{e}} / \tau, \hat{v}=v / v_{i}, v_{i}{ }^{2}=2 T_{i} / m_{1}, F_{M}$ is the local Maxwellian distribution function, $\Phi$ is the perturbed eiectrostatis potential, $A_{\|}$is the parallel component of the perturbed vector potential, and $H_{i}$ is related to the perturbed ion distribution $f_{i}$ by $f_{i}=-\left(e \phi / T_{i}\right) F_{M}+H_{i}$. Since we will work in the $1 \mathrm{imit} w_{t I}<w$, the $\theta$ dependence of $v_{k}$ and $v_{L}$ wili be Ignored.

In the following, we will employ the ballooning mode formalism which uses $\varepsilon \equiv 1 / \mathrm{n}$ as an expansion parameter to develop an asymptotic solution of Eq. (1). The perturhed quantities have short perpendicular and long parallel wave lengths and can be expressed by the elkonal representation

$$
\phi=\hat{\phi}(\theta, s, \sigma) \exp [-1 S(\theta, s) / \varepsilon]
$$

where $S$ describes the rapid eloss fleld variations and $B \cdot 7 S=0$. In the axisymmetric case, $S$ can be expressed as 


$$
\begin{aligned}
s(\theta, s) & =(m / n-q) \theta+\int k_{q}(s) d q \\
& =-\Delta r_{q} q^{-}\left[s \theta-\int k_{q} d s\right]
\end{aligned}
$$

where $k_{q}(s) / E$ is the radial wavenumber in $(\theta, q)$ coordinates. Insert Eq. (2) intn Eq. (1) and expanding in powers of $\varepsilon$, we find at lowest order

$$
\begin{aligned}
& \left\{\omega-1 \omega_{t 1} \hat{v}_{\|} \partial / \partial \theta+\omega_{D i}\right\} \hat{H}_{i} \\
& \left.=\left(e F_{M} / T\right\rangle_{i}, \therefore-\omega_{* 1}\left[1+n_{i}\left(\hat{v}^{2}-3 / 2\right)\right\}\right\} J_{0}\left\{\hat{\theta}-v_{\|} \hat{A}_{\|} / c\right) \\
& \quad \approx c_{i}
\end{aligned}
$$

where

$$
\begin{aligned}
& \omega_{D i}=2 \omega_{k_{e}}\left(\varepsilon_{n} / \tau\right)\left(\hat{v}_{i}{ }^{2}+\hat{v}_{\perp}^{2} / 2\right)\left(\cos \theta+\hat{s}\left(\theta-k_{q}\right) s i n \theta\right), \\
& J_{0}=J_{0}\left(b_{i}{ }^{1 / 2}\right), b_{i}=2 b_{0}\left(1+\hat{s}^{2}{ }^{2}\right) \hat{v}^{2} / \tau, b_{\theta}=\tau k_{0}^{2} o_{i}^{2} / 2,
\end{aligned}
$$

and

$$
\rho_{i}=v_{i} / \Omega_{i}
$$

In Eq. (4) we w111 choose $k_{q}=0$ so that the perturbations are centered at the outside of the torus. This cholce of $k_{q}$ is made because we have learned from numertcal experlence that the maxima of $\operatorname{Im}(w)$ occur at $k_{q}=2 \pi N(N=0,1 \ldots)$ for up-down synmetric equilibrium. Fquation (4) is defined over an infinite 
range in $\theta$ without periodfcity constraint. The boundary condition for $\hat{H}_{i}$ is that $\hat{H}_{i}$ decays sufftcienty fast as $|\theta| \rightarrow \infty$.

In the limit $w_{t i} \ll w$, Eq. (4) can be solved to yleld

$$
\hat{H}_{i}=\frac{r_{i}}{\omega+\omega_{D i}}+\frac{i \omega_{t i} \hat{v}_{i}}{\left(\omega+\omega_{D i}\right)^{2}} \frac{\partial G_{i}}{\partial \theta}-\frac{\omega_{t i}{ }^{2} \hat{v}_{H}^{2}}{\left(\omega+\omega_{D i}\right)^{3}} \frac{\partial^{2} G_{i}}{\partial \theta^{2}}+0\left(\frac{\omega_{t i}}{\omega}\right)^{3} .
$$

The ion density and current perturbations, $5 n_{i}$ and $j_{k i}$, can be calculated froin Eq. (5) and are given by

$$
\begin{aligned}
& \frac{\delta n_{i}}{\hat{N}}=-e \hat{\Phi} / T_{i}+\int \hat{H}_{i} J d_{0} d^{3} v
\end{aligned}
$$

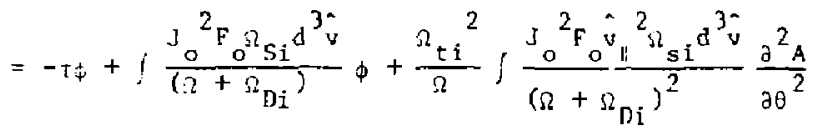

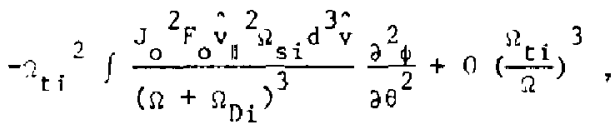

and

$$
\begin{aligned}
\frac{\partial}{\partial \theta} i_{\| j}= & \frac{\partial}{\partial \theta} \int e v_{11} \hat{H}_{i}{ }_{0} d^{3} v \\
= & \frac{N e v_{i}{ }^{2} t i}{i \Omega} \int J_{0}{ }^{2} F_{0} \hat{v}_{\|}{ }^{2} \Omega_{s i}\left(\frac{\partial^{2} A / \partial \theta}{\Omega+\Omega_{D i}}-\frac{\Omega \partial^{2} \phi / \partial \theta}{\left(\Omega+\Omega_{D i}\right)^{2}}\right) d d^{3} v \\
& +0\left(\frac{\Omega_{t i}}{\Omega}\right),
\end{aligned}
$$

where

$$
\begin{aligned}
& \phi=e \hat{\Phi} / T_{e}, A=\hat{e A} / T_{e}, \hat{A}=\int^{\theta} d \theta \hat{A}_{\| I} \omega v_{i} / i \omega_{t i} c, j i=\omega / \omega_{\star_{e}}, \eta_{D i}=\omega_{D i} / \omega_{*_{e}}, \\
& \Omega_{t 1}=w_{t 1} / w_{\hbar_{e}}, \Omega_{s 1}=\tau+1+\pi_{1}\left(\tilde{v}^{2}-3 / 2\right) \text { and } F_{0}=\left(1 / \pi^{3 / 2}\right) \exp \left(-i^{2}\right) \text {. }
\end{aligned}
$$


We will consider all the electrons to be highly circulating and collisions and trapped electron effects are not retained. The linearized gyrokinetic equation for electrons is given by

$$
\left[\Omega-i s \tau_{t e} \hat{v}_{\|} \frac{\partial}{\partial \theta}+\Omega_{D e}\right] \hat{H}_{e}=\left\langle e F_{M} / T\right)_{e} \Omega_{s e}\left(\hat{\phi}-v_{H} \hat{A}_{\|} / c\right) \equiv G_{e}
$$

where

$$
\begin{aligned}
& \Omega_{n e}=-T \Omega_{D i}, \Omega_{t e}=v_{e} /\left(q R_{\omega_{e}}\right), v_{e}^{2}=2 T_{e} / m_{e}, \\
& a_{s e}=\Omega-\left[1+\eta_{e}\left(\hat{v}^{2}-3 / 2\right)\right], \hat{v}=v / v_{e} \text {, and } \eta_{e}=d \ell n T e / d \ell n N .
\end{aligned}
$$

Since we are interested in the 1 fmit $\Omega \ll \Omega_{\text {te }}$, the $\theta$ dependence of $\vec{v}_{f}$ will be ignored and Eq. (B) can be solved with the asymptotic decaying boundary condition to give

$$
\hat{H}_{e}=j^{\theta} d \theta \frac{1 G_{e}}{\Omega_{t e} \hat{v}_{\|}}+\int^{\theta} d \theta^{\prime} \frac{\Omega+\Omega_{D e}}{\Omega_{t e} \hat{v}_{\|}} f^{\theta^{\prime}} d \theta^{\prime} \cdot \frac{G_{e}}{\Omega_{t e} \hat{v}_{\|}}+0\left(\frac{\Omega}{\Omega_{t e}}\right)^{3} .
$$

The electron density perturbation, on $n_{e}$, and current perturbacion, $f_{1}$, can be obtafned from Eq. (9) and are given by

$$
\operatorname{on}_{e} / N=\hat{e} / T_{e}+\int \hat{H}_{e} d^{3} v=\phi-\Omega-1 / \Omega A+0\left(\Omega / \Omega\left(\Omega_{t e}\right)^{2},\right.
$$




$$
\begin{aligned}
& \frac{\partial}{\partial \theta} j_{l e}=-\frac{\partial}{\partial \theta} \int e v_{\|} \hat{H}_{e} d^{3} v \\
& =-\frac{\operatorname{Nev}^{e}}{i \Omega_{t} e^{\Omega}}\left[\Omega(\Omega-1)(\theta-A)+2 E_{n}\left(\Omega-1-n_{e}\right) f_{\theta} A\right] \\
& +0\left(\frac{?}{x_{\text {te }}}\right)^{2},
\end{aligned}
$$

where $f_{e}=\cos \theta+\hat{s} \sin \theta$. Comparing F.q. (T) with Fq. (11) we note that $a j_{l i} / \partial j$ is of order $\left(\Omega_{t i} / \Omega\right)^{2}$ smaller than $\partial j_{t e} / \partial g$.

The basic set of coupled one dimensinnal equations governing the eigennodes of the system can now be written in terms of the solutions of the perturbed quantities given by Eqs. (7), (8), (10), and (11). These are thr quasi-neutrality condition and the parallel component of Ampere's law along 5 . The quasi-neutrality condition can be expressed as

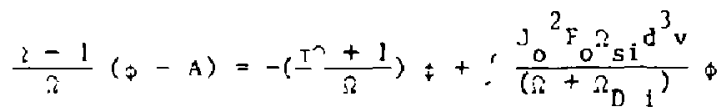

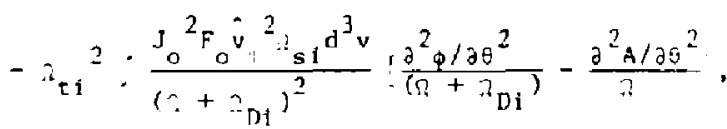

and the parallel Ampere's law is given by

$$
\begin{aligned}
& \frac{\partial}{\partial \theta}\left(1+\vec{s}^{2} \theta^{2}\right) \frac{\partial A}{\partial \theta}=\Omega_{A}^{-2}\left(\Omega(\Omega-1)(\phi-A)+2 \varepsilon_{n}\left(\Omega-1-\eta_{e}\right) F_{\sigma_{1}} A\right. \\
& -\Omega_{t 1}{ }^{2} \int \frac{\mathrm{J}_{0}{ }^{2} F_{0} \hat{\nu}_{\| 1}{ }^{2}{ }^{2} s i}{\left(\Omega+\Omega_{D 1}\right)}\left[\frac{\partial^{2} A}{\partial \theta}-\frac{\Omega}{\left(\Omega+\Omega_{0 i}\right)} \frac{\partial^{2} \phi ;}{\partial \theta^{2}} d^{3} v,\right.
\end{aligned}
$$

where $\lambda_{A}^{-2}=[B / 2(1+1 / \tau)]\left(q / \varepsilon_{n}\right)^{2}$ and $B=8 \pi N\left(T_{C}+T_{i}\right) / g^{2}$. Since the paralle $]$ 
electric field perturbarion $E_{\|}$is proportional to $a / \partial \theta(\phi-A)$, the right hand side of Eq. (12) represents the kinetic fon contributions from finite Larmor radius and magnetic drift resonance effects. If we further neglect terins of order $\left(\Omega_{t i} / \Omega\right)^{2}$, Eqs. (12) and (13) can be reduced to a single second order differential equation, l.e.,

$$
\begin{aligned}
\frac{\partial}{\partial \theta}\left(1+\hat{s}^{2} \theta^{2}\right) \frac{\partial A}{\partial \theta}=\Omega_{A}^{-2} & \left\{2 e_{n}\left(\Omega-1-n_{e}\right) f_{\theta}-s(\Omega-1)\right. \\
& \left.+(\Omega-1)^{2} /\left(1+\tau-\int \frac{J_{a}{ }^{2} F_{0} \Omega_{s i} d^{3} v}{\left(\Omega+\Omega_{D i}\right.}\right)\right\} A .
\end{aligned}
$$

Equation (14) describes the kfletic ballooning mode with even $A$ in $A$ and can he solved as an elgenvalue equation for $\Omega$ under the boundary condition that A Herays asymptotically as $|\theta|+\infty$. We can now compare Eq. (14) with the inalngons MID equations. ${ }^{2-5}$ If $a_{D i} / s t$ and $b_{\theta}$ are taken as mail parameters and terms of order $\left(\Omega_{D_{1}} / \Omega\right\}^{2}$ and $\left(b_{\theta}\left(1+s^{2} \theta^{2}\right)\right\}^{2}$ are neglected, then Eq. (14) redives $10^{6}$

$$
\begin{aligned}
\frac{1}{y+y}\left(1+s^{2} g^{2}\right) \frac{\partial}{\partial \theta} A= & -\Omega_{A}^{-2} \mid 2 \varepsilon_{n}\left(1+n_{e}+\left(1+n_{i}\right) / \tau\right] f_{\theta} \\
& +a l a+\left(1+n_{1}\right) / \tau \mid b_{\theta}\left(1+s^{2} \theta^{2}\right) ; A .
\end{aligned}
$$

In the limlt $b_{\theta}+0$, Eq. (15) corresponds exactly to the ideal, single-fluid MHD ballooning mode equation. From Eq. (15) we see that the elgenfrequency at marginal stability is given by $\Omega=-\left(1+n_{1}\right) / 2 \tau$, and the critical $\beta_{c}$ versus $b_{\theta}$ is shrom in Fig. (1) by solving Fa. (15) numerically for the parameters: $\dot{s}=n . r, E_{n}=0.2, q=2, T=1$, and $n_{e}=n_{1}=0$. Figure 1 clearly shows the 
Finite Larmor radius stablization of the MHD ballooning modes, In the Iimit $b_{\theta}+0, \beta_{c}$ approaches to the ideal MHD eritical $\beta$. However, the assumptions, $\Omega_{\mathrm{D} 1} / \Omega \ll$, and $\mathrm{b}_{\theta}\left(1+\mathrm{s}^{2} \theta^{2}\right) / \tau \ll 1$, that lead to Eq. (15) become invalid at marginal stability because the mode structure becomes very broad and extends to $\theta \sim 0\left(10^{2}\right)$. Therefore, the result in Fig. 1 is incorrect and we must employ Eq. (14) to study the ballooning modes near marginal stability.

II * RESULTS

Ir. this section we present the resules of an eigenmode analysis of kinetic ballooning mores (with the even A solution) by solving Eq. (I4) numerically. Let us Eirst consider the $n_{i}=0$ case. We observe that for $\Omega=$ - $1 / \mathrm{t}\left(\mathrm{i} . \mathrm{e} ., \omega=\omega_{\star i}\right), \mathrm{Eq} .(14)$ reduces exactly to the ideal MHD ballooning mode equation at marginal stability[2-5] (with $\Omega=0$ ), i.e.,

$$
\frac{\partial}{\partial \theta}\left(1+s^{2} \theta^{2}\right) \frac{\partial}{\partial \theta} A+\beta_{c}\left(q^{2} / \epsilon_{n}\right)\left[1+\eta_{e} /(1+1 / \pi)\right](\cos \theta+\sin \sin \theta) A=0 .
$$

Therefore, the ericical B obtalned from Eq. (16) is exactly the same as the ideal MHN $B_{C}$ and is independent of the kinetic ion contributions due to finite Larmor radius and magnetic drift resonance effects. Note that this conclusion of $B$ is unchanged when we employ the self-consistent equilibrium, and the second critical $\theta$ also occurs at $\omega=\omega_{\star_{1}}$. It is not difficult to understand this result physically because at marginal stabllity with $\Omega=-1 / \tau$, the 1 ons behave adiabatica1ly and the parallel electric field perturbation E vanishes Identically which corresponds to the Ideal MHD assumption. This phenomenon can also be seen In Ftg. 2 where the numerical eigenfrequencles from solving Eq. (14) are shown versus $B$ for the parameters: $\hat{s}=0.5, E_{n}=0.2, q=2$, 
$\tau=1, b_{\theta}=0.1$ and $n_{1}=n_{e}=0$. The brisken curve shows the growth rates of the ideal MHD balloonlng modes $\left(b_{g}+01\right.$ imlt $)$. We note that the g-owth rates of the klnetle ballooning modes are reduced due to fintte Larmor radius and magnetic irtft effects, but reach marglnal stablitity at the same $B$ as the ídeai MHI modes.

Note that in solving Eq. (14) we have made the approximation 8

$$
\nabla_{D i}=2\left(\varepsilon_{n} / \tau\right)\left(2 \hat{v}_{\|}^{2}\right) E_{\theta}
$$

so that the velocity space integral in Eq. (14) can be carried out analutically to yieid

$$
\int J_{0}^{2} F_{0} \Omega_{s i} d^{3} v /\left(\Omega+\Omega_{D i}=\left[\left(\tau \Omega+1-n_{i} / 2\right) r_{0}+n_{i} b\left(r_{1}-r_{0}\right)\right] D_{0}+n_{i} r_{0} D_{1}\right.
$$

where

$$
n_{0}=\left(\tau / 4 \varepsilon_{n} f_{\theta}\right) Z(\xi) / \xi_{2}, n_{1}=\left(\tau / 4 \varepsilon_{\pi} F_{\theta}\right)(1+\varepsilon Z(\xi)), \xi_{0}^{2}=-\left(\Omega \tau / 4 \varepsilon_{n} F_{0}\right),
$$

$z$ is the plasma dispersion functian, $r_{0,1}=I_{0,1}(b) \exp (-b)$ and $b=b_{\theta}$ $\left(1+\hat{s}^{2} \theta^{2}\right) / T$. In obtaining $D_{0}$ and $D_{1}$, we must perform the proder analytic continuation of the integration contour and $\xi$ in the complex $\hat{v}_{i}$ space. This approximation deforms the resonant surface in velocfty space into a plane without affecting the basic magnetic drift resonance effects.

In addition to the eigenmode (branch I) described in Fig. 2, there exists another eigenmode branch (branch II). For $\eta_{j}=0$, branch II remains stable for all 8 . Figure 3 shows the dependence of the elgenfrequency $\Omega$ on $\eta_{i}$ for 
these two branches of eigenmodes with parameters: $\eta_{e}=0, \pi=1.0, \varepsilon_{n}=0.2$, $b_{\theta}=0.1, q=2.0, \hat{s}=0.5$, and $B=0.017$. Branch I is unstable for small $n_{i}$ and becomes stable for large $\eta_{1}$. On the other hand, branch II 1s stable for small $\eta_{1}$, but becomes ilghly destabllized as $n_{1}$ increases. For both branches the real frequencies decrease as $n_{i}$ increases. Figure 4 shows the elgenfrequency $a$ versus $\eta_{e}$ for $\eta_{1}=0, T=1.0, \varepsilon_{n}=0.2, b_{0}=0.1, q=$ $2.0, \hat{s}=0.5$, and $\beta=0.017$. Here, contrary to the $n_{1}$ dependence in Fig. 4 , branch $I$ is destabilized by $n_{e}$ and branc. II remalns stabie for all $n_{e}$ shown. For both branches the real frequencies increase with $n_{e}$.

Figures $5(a)$ and $5(b)$ show the elgenfunctions $A(\theta)$ of branches I and II for $n_{e}=n_{j}=0, B=0.017, I=1.0, \varepsilon_{n}=0.2, b_{\theta}=0.1, \hat{s}=0.5$, and $q=$ 2.0. The ejgenfrequencies are $\Omega=-0.896+0.11$ i for branth i and $\Omega=-1.59$ - 0,073i for branch II. The modes are near marginal stability and have very broad structure. Since the ion magnetle drift frequency $\omega_{D 1}$ is secular in $\theta$, the perturbative treatment of $\omega_{D i}$ term breaks down for $\theta \geqslant\left(\tau / 2 \varepsilon_{n} s\right)=5$. 41 so the assumption $b_{\theta}\left(1+\hat{s}^{2} \theta^{2}\right) / \tau \ll 1$ is not valdd for $\theta \geq\left(\tau / b_{\theta} a^{2}\right)^{1 / 2} \approx 6$.

We have studied the behavior of these two branches of efgenmodes in the $\left(n_{e}, n_{1}\right)$ plane. We find that for the set of parameters deseribed in Figs. 3 and 4 there is a branch point at $\left(n_{1}, \eta_{e}\right) \circ(0.71,0.35)$ where these two branches colneide and become a double root with $\Omega \sim(-1.8,0.185)$. If we vary $\eta_{1}$ and $\eta_{e}$ along a closed path $1 n\left(n_{i}, n_{e}\right)$ plane a round the branch point once, we find that branch I interchanges with branch II.

For $n_{1} \neq 0$ the eritical $B$ is different from the ideal MHD $B_{c}$ and is usually smaller. The dependence of the eigenfrequency of the most unstable mode on $B$ is Jisplayed in Fig. 6 for $\eta_{I}=\eta_{e}=1.0, \hat{s}=0.5, \varepsilon_{n}=0.2, q=$ $2.0, b_{\theta}=0.1$, and $\tau=1.0$. The mode becomes marginaliy stable at. $B_{c}=0.006$ with eigenfrequency $\Omega=-4,0$. The gri: h rate of the ideal MaD made is also

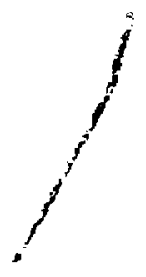


sinown in Fig. 6 fo: comparison. Figure 7 shows the dependence of id on by for $L=0.017$ and the other parameters are the same is in Fig. 6. The finite Lamor radius effect ean reduce the growth rate, but does not stahilize the mode even when $b_{\theta}$ is larger than unity. The real frequency derreases as $b_{0}$ increases. For $b_{\theta} \leq 10^{-3}$, the condition $\left(\omega_{t j} / \omega\right) \ll 1$ is not well satisfied and the solutcons are questionahle.

The evolution of the ballooning modes is alsa examined as the parameters, sind $\varepsilon_{n}$, arc varied. The fixed parameters are $n_{e}=n_{i}=1,0, \beta=0 . n 2, b_{9}=$ $0.1, q=2.0$, and $\tau=1.0$. In Fig. 8(a) (with $s_{l}=0.2$ ) the shear trabilization of the bailooning modes ocsurs at $\bar{s}=1.15$. Both the real

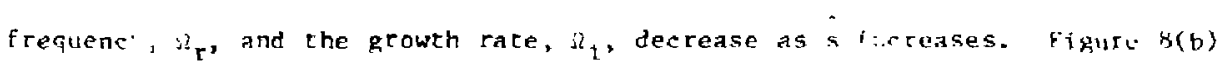
Jisplays the dependence of id an $\varepsilon_{n}$ with $\hat{s}=0$. 5. There are two stability boundaries with $\varepsilon_{n]}=0.0135$ and $E_{+1} 2=0.306$. For small $r_{n}\left(\left|\omega_{D i}\right| \omega \mid \ll 1\right)$ the magnetir drift resonance effect is exponentially small and the nodes evolve from a pair of almose complex conjugates into two real s? solutions as fn decreases helow $c_{n l}$. This differs from the ideal MHD resulcs in that there is only one critical $\varepsilon_{n}$ for a low $B$ equilibrium with roncentrir, eirenlar magnetic surfaces.

\section{CONCIUSIONS}

In chis paper we have employed the high n ballooning mode formalisin to derive the efgenmode equation for kinetic ballooning modes of arbitrary wavelengths in the limbt $w_{t i}<w<w_{t e}$. The lon magnetic drift frequency, $\omega_{D_{L}}$, is not taken to be smaller than the mode flequency, $\omega_{\text {s }}$ so chat the ion ragnetic drift resonances are retained. In the $11 \mathrm{mits} \omega_{D i} / \omega \ll 1$ and $b_{\theta} \rightarrow 0$, the eigenmode equation reduces to the ideal MHD ballooning mode equácion. The 
eigenmode equation is then analyzed numertcally by using a shooting rode. Two branches of elgenmodes at - found to exist. For $\eta_{1}=0$ we have observed that the eritical $B$ of branch $I$ which occurs at $\omega=w_{\hbar_{i}}$ is identical to the ideal MHD $\beta_{c}$ and is independent of the Finite Larmor radius and magnetirs drits rosorice effects. Branch II remains stable for all $B$ at $n_{1}=0$, hut can he more unstable than branch $I$ as $n_{I}$ is varied. For $n_{1} \neq 0$ the numerical results have shown that the critical $B$ is smaller than the ideal MHD $B_{C}$ and the finite Larmor radius effect can reduce the growth rate, but does not stabilize the ballooning modes. The ton magnetic drift resonance effect is destabilizing. The denendence of $z$ on $E_{n}$ shows that there are two stability boundaries in ${ }^{n}$. This is different from the ideal MHD predirtion of only one critical s $n$ for a low : equilibrium with concentric, circular magnetic surfares.

Finally, this work is incomplete since kfnetic effects, sic'l as trapp rd particles, Landau resonances, and collisions 9,10 , are suppressed here in order to concentrate on the finte ion Larmor radius and ion marnetc drift resonance effects. The self-consistent MHD equilibrium nust also be included before we can determine the critical $B$ of tokamaks.

\section{ACKNOWLEDGMENTS}

The authot thanks Dr. M. S. Chance for useful discussions.

This work is supported by the United States Department of Energy contract No. DE-ACO2-76-CHO3073. 


\section{REFERENCES}

1. D. Dobrott, D. B. Nelson, J. M. Greene, A. H. Glasset, M. S. Chance, and E. A. Frleman, Phys. Rev, Lett, 39, 943 (1977).

2. A. H. Glasser, Proceeilings of the Finfte Beta Theory Workshap, Vinenna, 1977, edited by B. Coppi and W. Sadowskt, (U. S. Dept. of Fnergy, Conf$7709167,1977)$, p. 55.

3. Y. C. Lee and J. W. Van Dam, Varenna, 1977 (as in ref. 2), p. 93.

4. 3. H. Connor, R. J. Hastle, and J. B. Feglor, Proc. R. Soc. Land. A 365, I $(1979)$.

5. R. L. Dewgr, M. 5. Chance, A. H. Glasser, J. M. Gzeene, and F. A. Frifeulan, PPPL-1587 (1979).

6. M. S. Chu, C. Mhu, G. Guest, J. Y. tisu, and T, Ohkawa, Phys. Rev. Lett. 41. 247 (1978).

7. K. T. Tsang, ORNL/TM - 7324 (1980).

8. C. Z. C'eng and K. T. Tsang, PPPL-1761 (1981).

9. W. M. Tang, J. W. Connor, and R. J. Hastie, Nucl. Fusion 20, 1439 (1980).

10. T. M. Antonsen and B. Lane, Phys, Fluids 23, 1205 (1980). 
\# 31 T0083

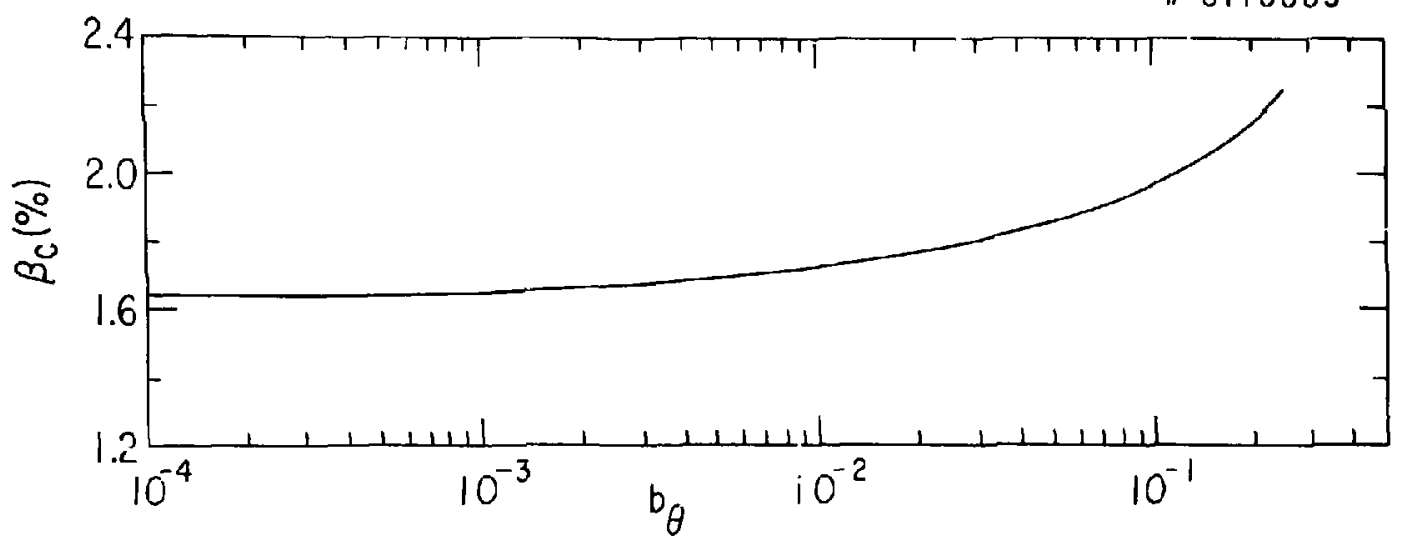

Fig. I Critical $\beta$ versus $b_{\theta}$ for $\hat{s}=0.5, \varepsilon_{n}=0.2, q=2.0, T=1.0$, and $\eta_{i}$

$=n_{e}=0$ by solving Eq. (15). 


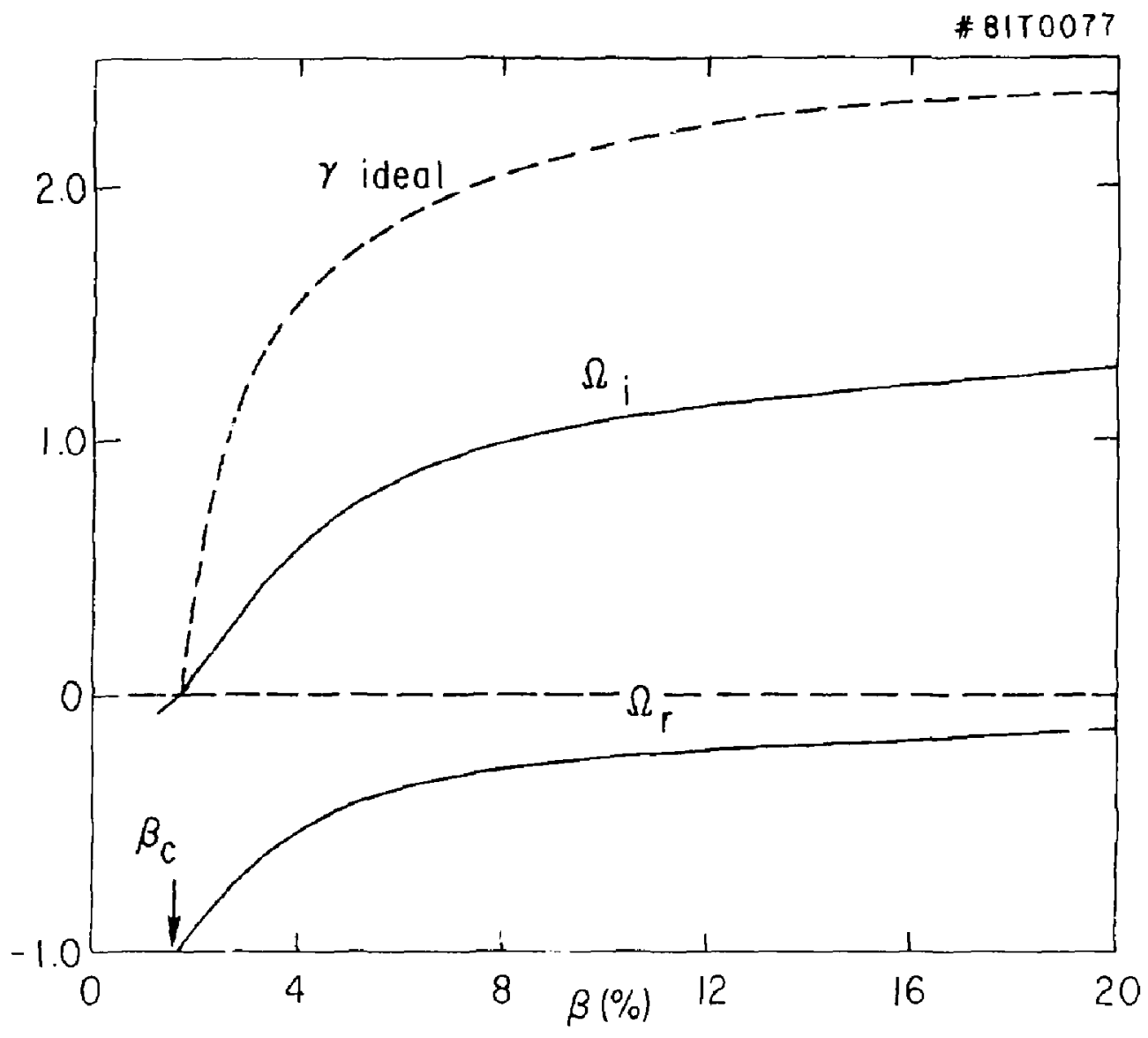

Fig. 2 The dependence of $\Omega$ on $B$ for $a_{\hat{u}}=0.1, \hat{s}=0.5, \varepsilon_{n}=0.2, q=2.0$, $\mathrm{i}$ $=1.0$, and $n_{e}=n_{1}=0$. The 1 deal MHD gruith rate is also shown. 


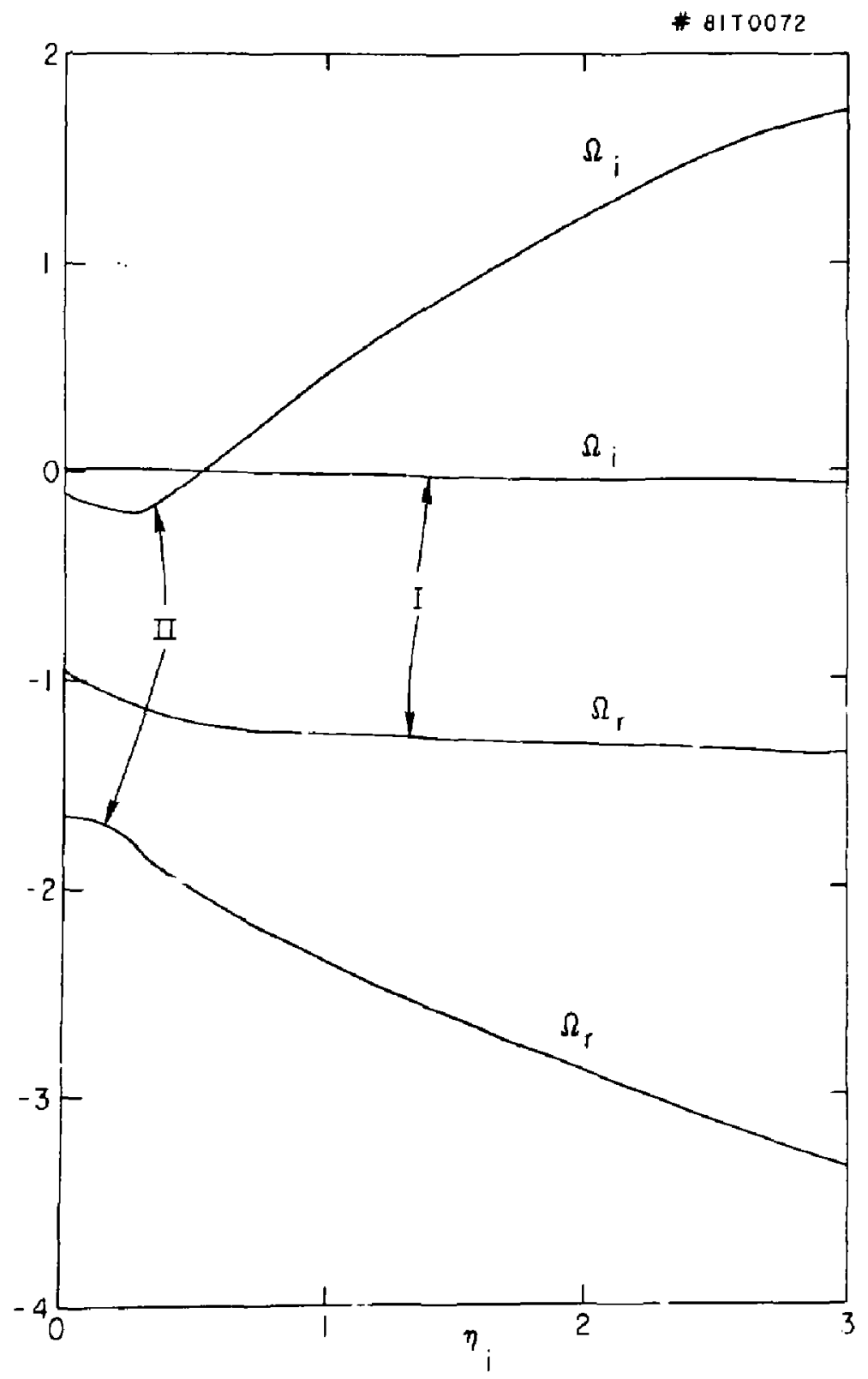

F18. 3 The dependence of $\Omega$ on $n_{1}$ for two branches of eigenmodes. The fixed parameters are $\beta=0.017, b_{\theta}=0.1, \hat{s}=0.5, E_{n}=0.2, q=2.0, t=$ $1, r_{1}$ and $r_{t}=0$. 
8170071

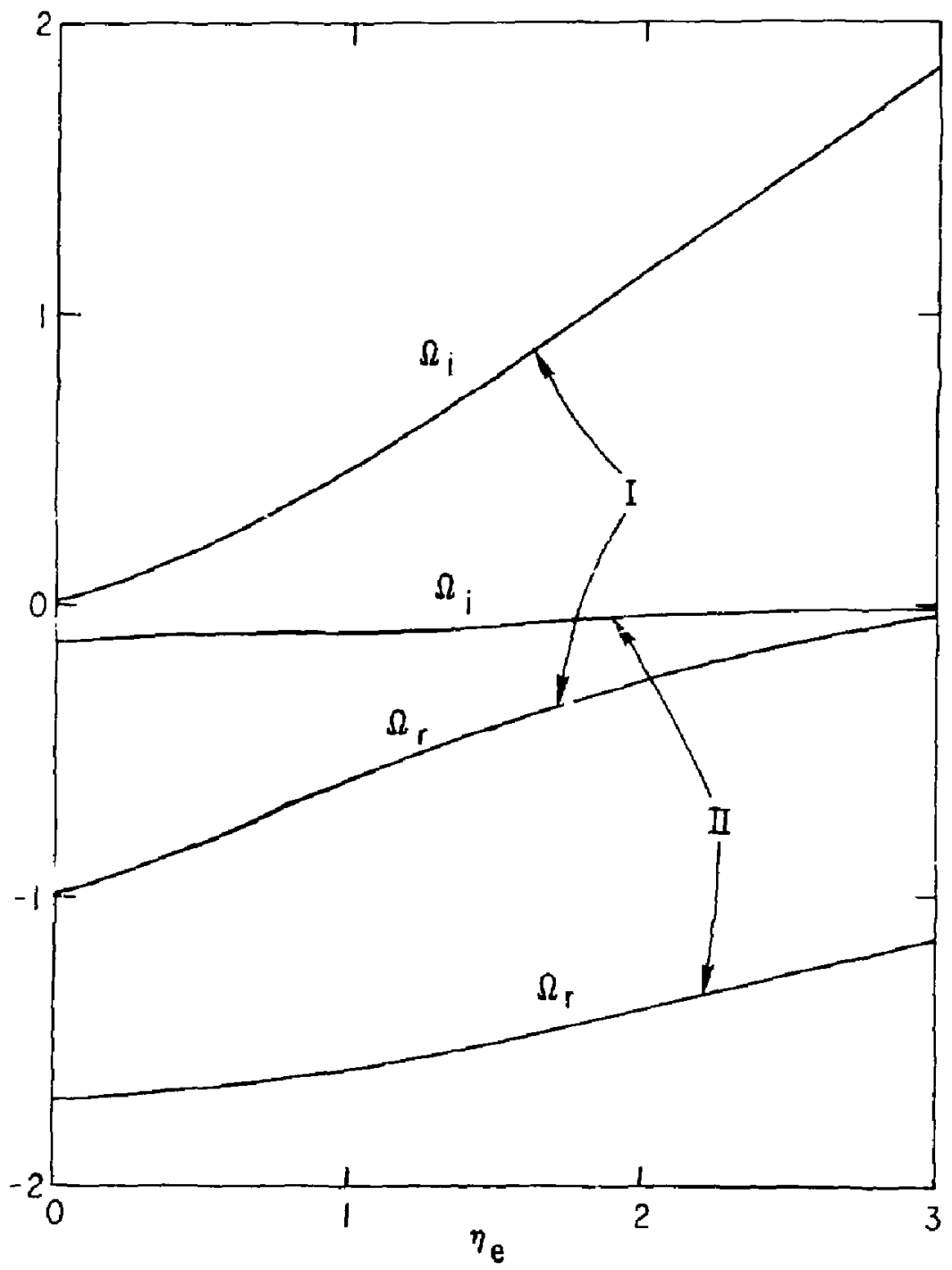

Fig. 4 The dependence of $\Omega$ on $n_{e}$ for two pranches of eigenmodes with $n_{i}=$ D. The other parameters are t!e same as in Fig. 3. 
$-20-$
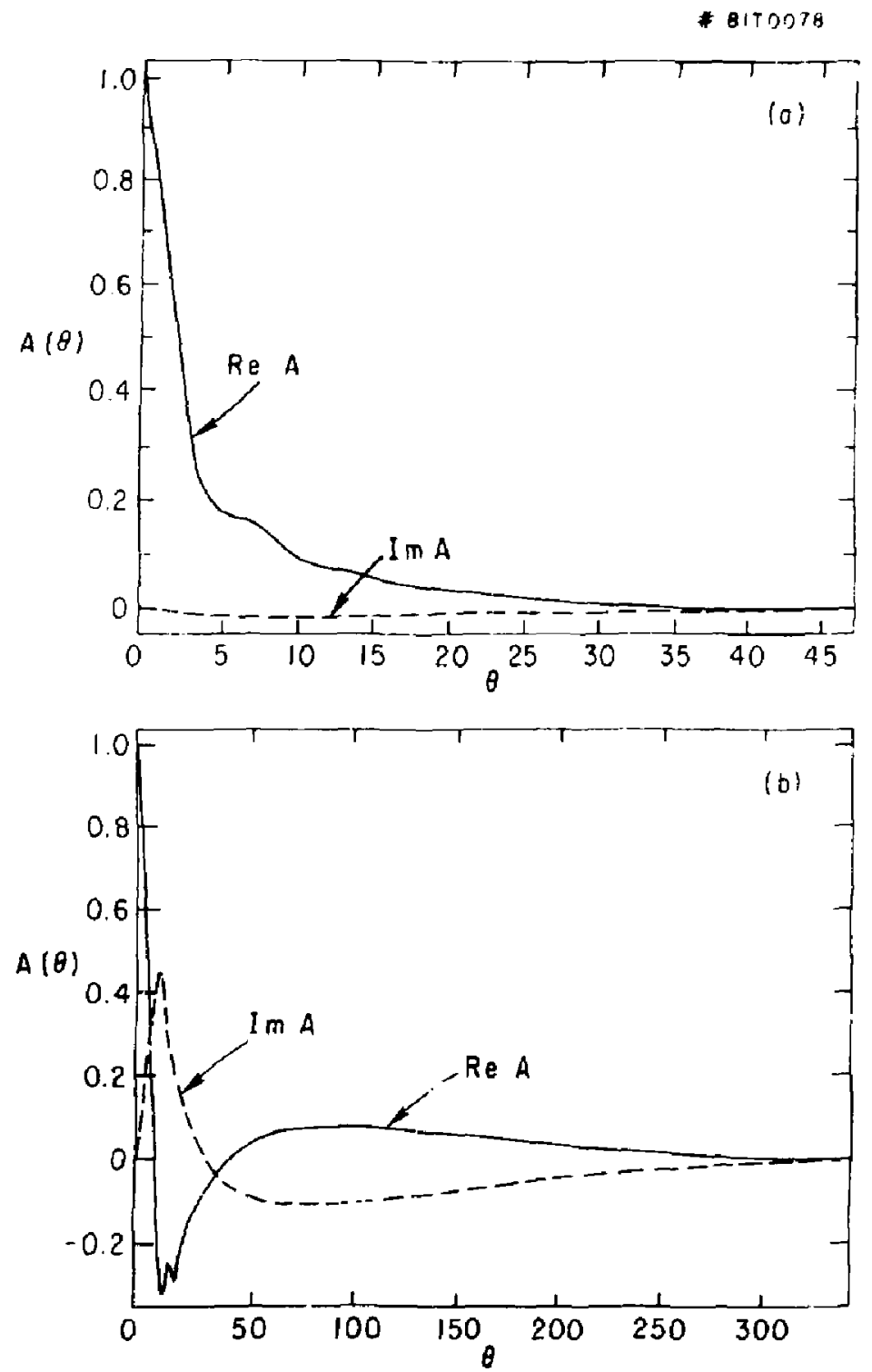

Fig. 5 Plot of the elgenfunction of two branches of elgenmodes (for $\beta=$ $0.02, b_{\theta}=0.1, \hat{s}=0.5, E_{n}=0.2, q=2.0, \tau=1.0$, and $\eta_{e}=\eta_{i}=$ $0)$ : (a) branch I with $\Omega=(-0.896,0.11)$, (b) branch II with $\Omega=(-$ $1.588,-0.073)$. 
\# 81T0084

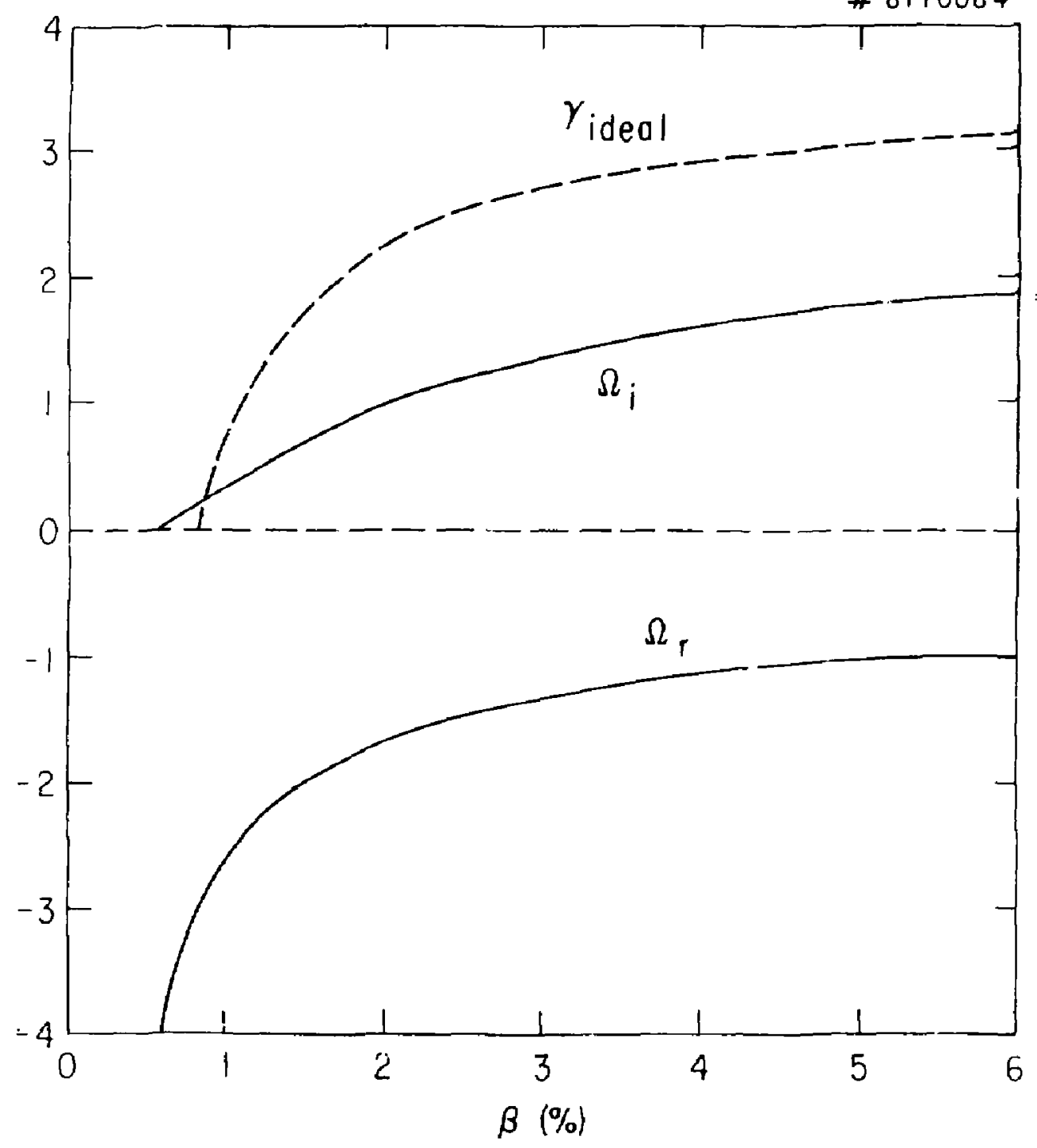

Fig. $b$ The dependence of $\lambda$ on $B$ for $b_{G}=0.1, \hat{s}=0.5, \varepsilon_{n}=0.2, \eta=2.0,1$ $=1.0$ and $n_{e}=n_{i}=1.0$. The corresponding ideal Mls growth rate is also shown. 
\#81T0076

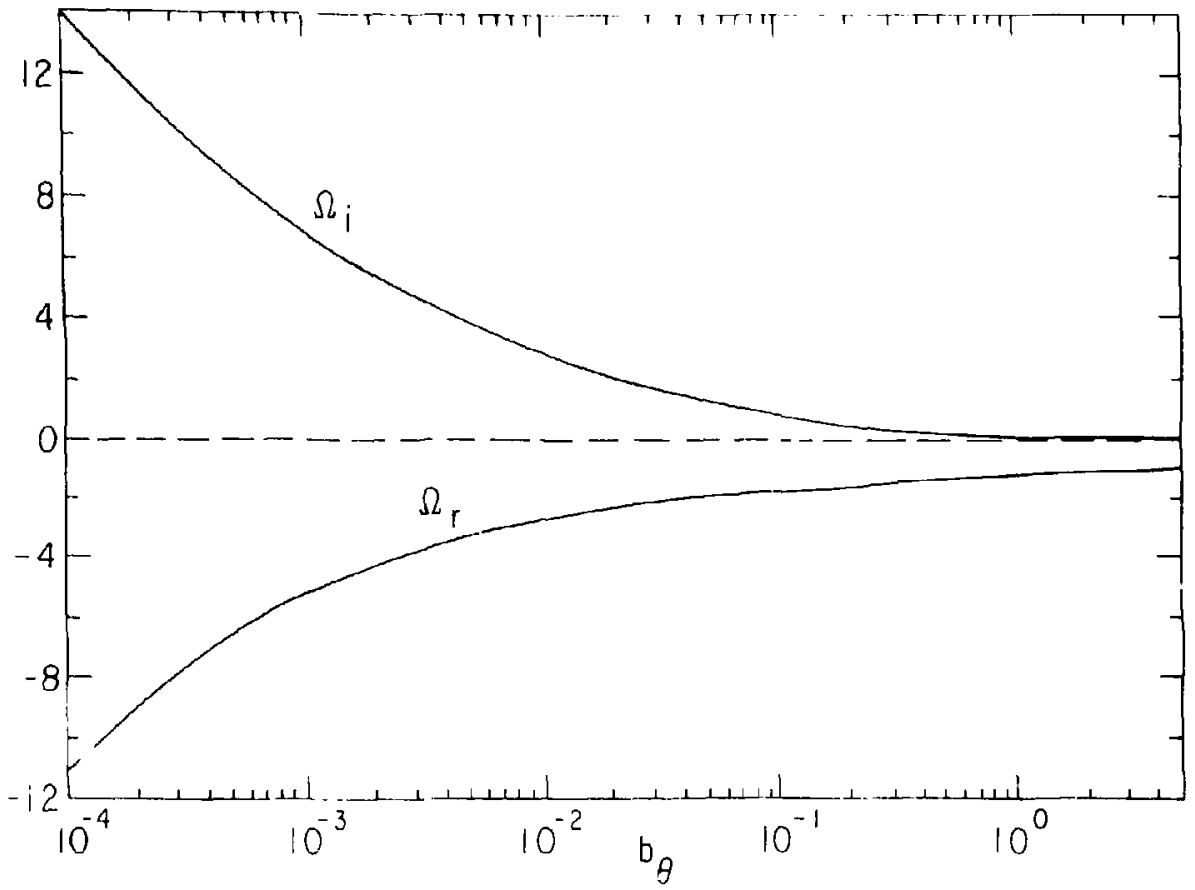

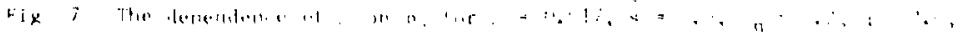

$=1,1,1,1,1,1$. 
$-\therefore 1-$

* B170075
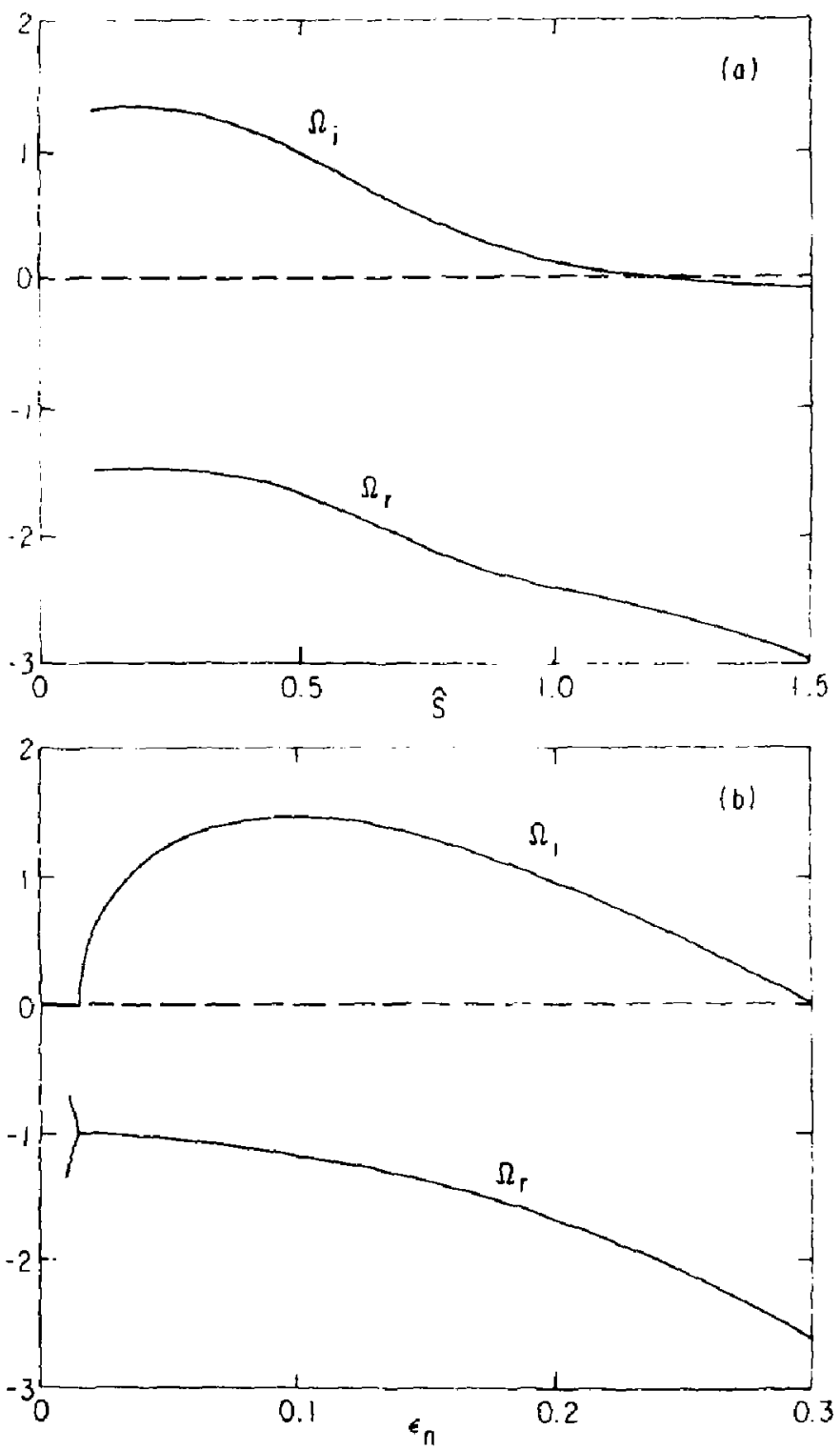

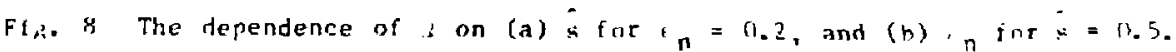
The $f$ txed parameters are $B=0.02, b_{B}=\cdots .1, i=1.0, q=2.0$, and $n_{e}$ $=n_{1}=1.0$. 